\title{
Absolute Quadrant Determinations from Speckle Observations of Binary Stars
}

\author{
W. G. BAGNUOLO, B. D. MASON, D. J. BARRY, W. I. HARTKOPF, \\ \& H. A. McALISTER \\ Center for High Angular Resolution Astronomy, Georgia State University, \\ Atlanta, GA 30303, USA
}

\begin{abstract}
Reduction of speckle data obtained for binary stars is typically carried out using power spectrum or, equivalently, autocorrelation methods. An especially powerful algorithm from which accuate differential astronomy can be obtained is the vector autocorrelation technique. While such methods are highly suited to extracting astrometric information from very large volumes of speckle data in near real-time, they inherently introduce a $180^{\circ}$ ambiguity in position angle measurement. We briefly summarize results with a new algorithm which maintains most of the simplicity of vector autocorrelation while removing the quadrant ambiguity.
\end{abstract}

True position angle quadrant determination is difficult in stars with a small magnitude difference. This difference is position can result in errors of many physical parameters, mass and distance among them. The approach of using the autocorrelation function has as a side effect an inherent $180^{\circ}$ ambiguity in the quadrant.

The directed vector-autocorrelation or DVA retains both the $x$ and $y$ position of each pixel as well as the direction, pixel to pixel from brighter to dimmer. The resulting DVA "autocorrelogram" for a binary star produces "center", "principal", and "ghost" spots, and the true position angle is given by the position of the principal spot relative to the center spot. Analysis software has been developed for extraction of these spots and an estimate of the SNR for quadrant determination. In Bagnuolo et al. 1992 we describe the method as applied to binaries with "known" and "unknown" quadrants. To date, true quadrants for about 100 stars have been determined. A calcite crystal and polarizing filter inserted into the optical path of our speckle camera have been used to create artificial binary star speckle patterns to test this algorithm (Bagnuolo et al. 1990, Barry et al. this Colloquium, Mason et al. 1992). According to the results presented in the latter paper, we can routinely detect binary componets for $6^{\text {th }}$ magnitude stars for $\Delta \mathrm{m} \leq 3.5$ and determine quadrants for $0.2 \leq \Delta \mathrm{m}$ $\leq 3.0 \mathrm{mag}$. The algorithm can also produce estimates of $\Delta \mathrm{m}$ to about $0.2 \mathrm{mag}$ accuracy.

\section{REFERENCES}

Bagnuolo, W.G., Jr., Barry, D.J., Mason, B.D., \& Dombrowski, E.G. 1990, Proceedings of the SPIE on Amplitude and Spatial Interferometry, J. Breckinridge, ed., 1237, 286

Bagnuolo, W.G., Jr., Mason, B.D., Barry, D.J., Hartkopf, W.I., \& McAlister, H.A. 1992, $A J, 103,1399$

Mason, B.D., McAlister, H.A., Hartkopf, W.I., \& Bagnuolo, W.G., Jr. 1992, AJ, submitted 